\title{
Im Zeichen der Qualität
}

Prof. Dr. med. Hans Ulrich Rothen, Dr. med. Roger Lussmann, Chantal Britschgi, Ursula Betschart

Mitglieder der Schweizerischen Gesellschaft für Intensivmedizin

Vom 21. bis 23. September fand in Montreux die diesjährige Jahrestagung der Schweizerischen Gesellschaft für Intensivmedizin (SGI) statt. Wie bereits 2015 trafen sich auch in diesem Jahr wieder Experten aus unterschiedlichsten Disziplinen und zahlreiche Interessierte, um sich an einem Management-Seminar für leitende Angestellte auf Intensivstationen einen Tag lang gemeinsam mit Themen zu beschäftigen, die nicht nur für den Erfolg der Intensivmedizin wichtig sind, sondern vielleicht sogar für den des gesamten schweizerischen Gesundheitssystems: Das ICU Management-Seminar 2016 stand ganz im Zeichen der Qualitätssteigerung.

\section{Wenn Fehler passieren}

Effektives Qualitätsmanagement beginnt oftmals mit dem Eingeständnis, dass selbst bei höchsten Standards Fehler eben manchmal passieren: «Auch in einem hochkomplexen System wie der Intensivmedizin steht ein Mensch, dessen Fähigkeiten und Ressourcen begrenzt sind, im Zentrum», meint Andreas Valentin von der Europäischen Gesellschaft für Intensivmedizin (ESICM). Patientensicherheit besteht deshalb nicht nur darin, Fehler um jeden Preis zu vermeiden. Genau diese allenfalls zu defensive Haltung kann ebenfalls zu Fehlern führen. Wichtig ist, wie mit Fehlern umgegangen wird, wenn sie denn auftreten. Nur wer eine kommunikativ offene Fehlerkultur fördert, die alle Mitarbeiter - egal ob Chefarzt oder Pflegende - miteinbezieht, menschliche Limitationen anerkennt und die Komplexität des Umfelds reduziert, sorgt für eine effektive Qualitätsverbesserung.

\section{Mit externen Gutachten die eigene Arbeit hinterfragen}

Auch eine kritische Beurteilung durch Aussenstehende kann dazu führen, dass die Behandlungsqualität auf einer Intensivstation steigt. In einer sogenannten Peer Review erstellt eine externe und gerne auch interprofessionelle Expertengruppe ein Gutachten einer Station, wobei eine Vielzahl von Kriterien berücksichtigt wird. Für Hans Ulrich Rothen vom Inselspital Bern muss das Gutachten an sich nicht einmal im Vordergrund stehen. Alleine die Vorbereitung auf eine solche, etwa durch die Initiative Qualitätsmedizin (IQM) durchgeführte Beurteilung kann schon zu einer Qualitätssteigerung beitragen. Auch für die Experten kann die Erfahrung einer Peer Review von grossem Wert sein - schliesslich lernen diese dadurch die Arbeit und Prozesse einer anderen Abteilung und Klinik besser kennen.

Wie Rosmarie Wyss vom Inselspital Bern kann auch Anne-Claude Griesser vom CHUV in Lausanne, wo im letzten Jahr eine erste Peer Review durch IQM durchgeführt wurde, solche Gutachten empfehlen.

Peer Reviews sind eine gute Möglichkeit, um die Qualität der eigenen Arbeit einzuschätzen und für die Zukunft genauere Ziele zu definieren.

Peer Reviews steigern den interprofessionellen Fortschritt - eines der Kernanliegen der SGI -, weil Ärzteschaft und Fachpflege so noch enger zusammenarbeiten. Peer Reviews sind eine gute Möglichkeit, um die Qualität der eigenen Arbeit einzuschätzen und für die Zukunft genauere Ziele zu definieren.

Ursprünglich wurde das Peer-Review-Verfahren von IQM für das deutsche Gesundheitssystem konzipiert. Vor der Einführung in der Schweiz mussten deshalb einige Anpassungen vorgenommen werden. Wie Pascal Besson von $\mathrm{H}+$, der Vereinigung schweizerischer Krankenhäuser, betont, habe man besonders darauf wertgelegt, dass die Interprofessionalität überall gewährleistet ist und auch Pflegende als Experten eingesetzt würden.

\section{Mit Talentförderung für die Zukunft gerüstet}

Qualitativ hochwertige Intensivmedizin erfordert hochqualifizierte Mitarbeiter. "Aufgrund des drohenden Fachkräftemangels wird der Mitarbeiterförderung in 
Zukunft eine noch bedeutendere Rolle zukommen. Hier können Spitäler noch etwas von der Wirtschaft lernen", ist sich der emeritierte Ökonomieprofessor Norbert Thom sicher. Nachwuchsförderung scheitert in Kliniken häufig nicht am Talent, sondern am Vorgesetzten. Deren mangelndes Engagement - nicht selten ist daran eine Überbelastung schuld - ist häufig die entscheidende Schwachstelle in der klinikinternen Talentpflege. Traineeprogramme, wie sie in der Wirtschaft seit mehreren Jahrzehnten etabliert sind, lassen sich auch im Spital implementieren, um sogenannte High Potentials für sich zu gewinnen, zu entwickeln - nicht nur zu Führungs-, sondern auch zu Fachkräften - und im besten Fall auch zu binden. Zudem sagt Thom: «Ohne erheblichen Einsatz, auch von ganz oben, etwa vom Verwaltungsrat, sind solche Programme nicht durchzuführen.» "Gute Mitarbeiter verlassen nicht Institutionen, sondern Personen», meint Walter Hagmann von der FHS St. Gallen. Es gibt einige Erfolgsfaktoren bei der Förderung besonders talentierter Mitarbeiter.

\section{Nachwuchsförderung scheitert in \\ Kliniken häufig nicht am Talent, sondern am Vorgesetzten.}

Der Aufbau einer Vertrauenskultur ist hierbei einer der entscheidendsten. Dazu gehört auch das Gewähren gewisser Handlungsspielräume. Gerne spricht man hier auch von Empowerment, der Übertragung von Verantwortung, die entscheidend ist, damit High Potentials sich auch selbständig weiterentwickeln und über sich hinauswachsen können.

Aus dem Publikum geben Stimmen zu bedenken, dass ein solches aus der Wirtschaft übernommenes Programm zur Talentförderung in der Medizin schwierig umzusetzen ist, weil es in einer Klinik nur eine begrenzte Anzahl an Kaderpositionen gibt. Talente müssen sich schon deshalb extern nach neuen Chancen und Aufgaben umsehen. Aus diesem Grund kann man High Potentials in der Medizin gar nicht dazu bewegen, zu bleiben. Für Christoph Haberthür von der Klinik Hirslanden in Zürich ist genau diese Beförderung von eigenen Mitarbeitern in andere Kliniken ein grosser Erfolg für die ausbildende Station, weil sie ein Zeichen für die Qualität der eigenen Arbeit ist.

Bei der Talentförderung in einem interprofessionellen Umfeld wie der Intensivmedizin dürfen die Pflegenden nicht vergessen gehen. Dabei ist es besonders wichtig, so Mario Desmedt von der Fondation Asile des Aveugles, dass die Arbeit der Pflege auch von anderen Berufsgruppen als bedeutend eingestuft wird, dass die Beiträge geschätzt und anerkannt werden. Zudem sollte man dem Pflegepersonal laut Irene Penker vom Univer-
sitätsSpital Zürich ein gewisses Mitspracherecht bei der Zuteilung der Schichten einräumen - auf diese Weise wird eine Einrichtung zum Anzugspunkt, zum «Magnet Hospital» für hochqualifiziertes Pflegepersonal.

\section{DRG - ein System mit Weiterentwick- lungspotential}

Ohne Geld geht in der Intensivmedizin nichts, und ohne Diagnosis Related Groups (kurz DRGs) gibt es kein Geld. Zwar wird mit diesen DRGs versucht, das Abrechnungssystem national zu harmonisieren, trotzdem rechnet nicht jede Intensivstation ihre Leistungen gleich ab. Und genau hier liegt eines der Probleme von DRGs, meint Hervé Guillain vom CHUV. Weil keine ICU und kein Spital in der Schweiz gleich ist, gestaltet es sich schwierig, eine Abrechnungsmethode zu entwickeln, die für alle funktioniert. Für Simon Hölzer von SwissDRG gibt es noch Weiterentwicklungspotential. Denn obwohl bereits ziemlich viel vereinheitlicht wurde, besteht noch eine gewisse Heterogenität, etwa bei der Datenerfassung. «Das macht die Systementwicklung natürlich nicht gerade einfach", so Simon Hölzer.

Die akkurate Leistungserfassung in der Intensivmedizin wird vor allem dadurch erschwert, dass 10 Prozent der Langzeitpatienten satte 50 Prozent der Ressourcen beanspruchen und der Aufwand für diese kleine Gruppe kritisch kranker Patienten mit DRGs noch zu wenig genau erfasst werden kann, ist Jukka Takala vom Berner Inselspital überzeugt. Genau daran will Simon Hölzer mit SwissDRG im Dialog mit den Fachleuten zukünftig vermehrt arbeiten - damit Intensivstationen in der Schweiz sich besser vergleichen lassen, ein Benchmarking erleichtert wird und ICUs so auch den Klinikleitungen besser zeigen können, was sie zum Gesamterfolg des Spitals beitragen, wie Jérôme Pugin vom HUG in Genf sagt.

Für Ursula Betschart vom Kantonsspital St. Gallen ist die wirkungsvollste Art der finanziellen Absicherung eine hohe Qualität der eigenen Arbeit. Denn «zufriedene Kunden kommen wieder». So rät auch Conrad E. Müller von der Klinik Hirslanden in Zürich dazu, sich nicht allzu sehr in Zahlen zu verlieren: «Wir sollten nicht zu viel messen, sondern stattdessen Messsysteme vereinfachen.» Ansonsten verliert man eventuell diejenigen aus den Augen, die zu jedem Zeitpunkt im Zentrum stehen sollten: den kritisch kranken Patienten und seine Angehörigen.

Mehr zu den Bestrebungen der SGI, die Qualität in der Intensivmedizin zu steigern, lesen Sie im Beilageblatt zum Minimalen Datensatz der SGI (MDSi) unseres Flyers kritisch krank? . Der Flyer ist verfügbar unter www.sgi-ssmi.ch/index.php/broschueren.html 